\title{
Discovery of the first macrolide antibiotic binding protein in Mycobacterium tuberculosis: a new antibiotic resistance drug target
}

\section{Dear Editor,}

The prevalence of multidrug-resistant Mycobacterium tuberculosis (M. tuberculosis) is an increasing problem worldwide (Zumla et al., 2013; Dong et al., 2015). According to a 2014 World Health Organization (WHO) report, 480,000 individuals world-wide developed multidrug-resistant tuberculosis (MDR-TB) and more than 100 countries have cases of extensively drug-resistant tuberculosis (XDR-TB). Compared with drug-susceptible TB, MDR-TB and XDR-TB require prolonged therapeutic treatment with a combination of a number of second-line drugs (Chen et al., 2013). For patients where TB remains persistent despite prolonged therapy with second-line TB drugs, the add-on agents including bedaquiline and delamanid are recommended for salvage therapy (Günther, 2014; WHO, 2014; Shim and Jo, 2013).

The mechanisms of antibiotic resistance developed by bacteria are highly diverse (Alekshun and Levy, 2007), including protection of the antibiotic target by site-of-action mutations or by chemical modification of the target/target site (e.g., methylation in the ribosomal 23S rRNA by ErmMT methyl-transferase) (Buriankova et al., 2003). The direct modification or inactivation of antibiotics by specific enzymes (e.g., acetyltransferases, phosphotransferases) can be another resistance mechanism. Alternatively, reduced permeability or increased efflux (Black et al., 2014) of the drugs can also occur, thus preventing the drug from gaining access to the target. M. tuberculosis has adopted several of these strategies to become resistant to the most widely used antibiotics. One of these is the utilization of the ATP-binding cassette $(A B C)$ transporters, which are drug efflux pumps that lower the concentration of antibiotics within the bacterium. Indeed, $A B C$ transporters have been divided into three classes based on phylogenetic analysis (Dassa and Bouige, 2001): (i) the classical exporters (ii) importers which are composed of hydrophobic transmembrane domains and hydrophilic nucleotide-binding domains, and (iii) the type-II $A B C$ proteins that lack transmembrane domains (Nunez-
Samudio and Chesneau, 2013), some of which mediate antibiotic resistance (Kerr et al., 2005; Sharkey et al., 2016).

Bioinformatics analysis shows that $M$. tuberculosis possesses as many as $87 \mathrm{ABC}$ containing proteins. Amongst these, Rv3197 is predicted to be a non-canonical ABC protein with an N-terminal extension (1-117), an ABC1 motif (118-232) and an aminoglycoside phosphotransferase (APH) motif (256-312) but lacking any transmembrane regions. The sequence characterization suggested Rv3197 might be an antibiotic transporter. In our preliminary study, we evaluated the effect of Rv3197 in antibiotic susceptibility by measuring the MICs of several antibiotics including isoniazid, rifampicin, erythromycin, streptomycin, ampicillin, chloromycetin and tetracycline by overexpression of Rv3197 in Mycobacterium smegmatis ( $M$. smegmatis), which is commonly used in lab as a model bacteria for $M$. tuberculosis. Our results indicated that Rv3197 affected the susceptibility of erythromycin (Table S1).

Since a biosafety level 3 lab is needed to direct study of M. tuberculosis and the amino acid sequence of Rv3197 in M. tuberculosis is equivalent to Mb3320 in M. bovis BCG, a vaccine strain with low pathogenicity, we chose $M$. bovis BCG as a model for studying the function of Rv3197. Quantitative real-time PCR (qRT-PCR) analysis showed that the transcription level of $r 3197$ increases by 3.6 fold when $M$. bovis BCG is treated with $6 \mathrm{mg} / \mathrm{L}$ erythromycin (Fig. $1 \mathrm{~A}$ ), showing that expression of $r 3197$ is erythromycin-inducible. To verify the effect of Rv3197 on erythromycin susceptibility, we first made an Mb3320-deletion mutant strain, denoted $\Delta r$ 3197-BCG. This mutant strain grew at a similar rate to the wild-type strain in $7 \mathrm{H} 9$ medium (Fig. S1A), demonstrating that, under the conditions where no antibiotic is added, Rv3197 has no effect on cell growth. However, upon exposure to $2 \mathrm{mg} / \mathrm{L}$ of erythromycin, cell growth was reduced in $\triangle r v 3197-B C G$ compared to wild-type BCG. This effect was partially reversed in a complemented pMV361-rv3197/ $\Delta r$ 3197-BCG strain (Fig. 1B). In the presence of $3 \mathrm{mg} / \mathrm{L}$ of erythromycin, $M$. smegmatis, which contains the rv3197 


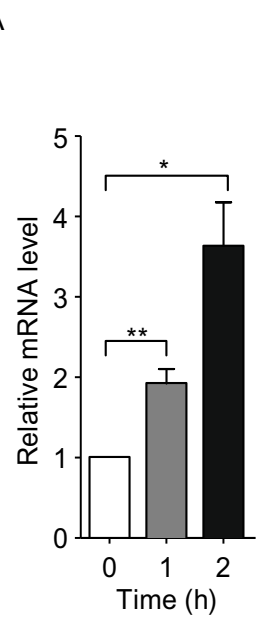

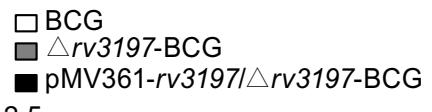

C

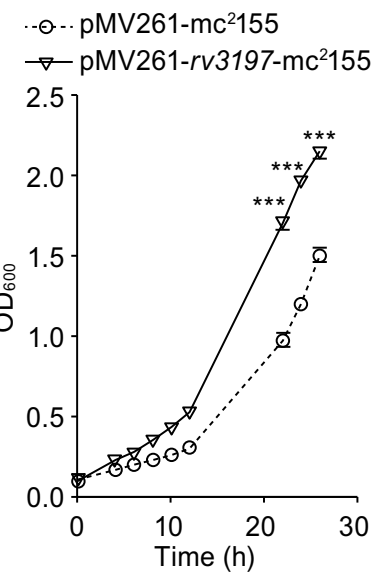

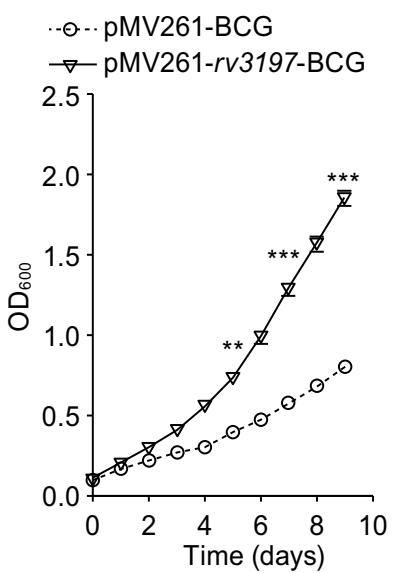

E

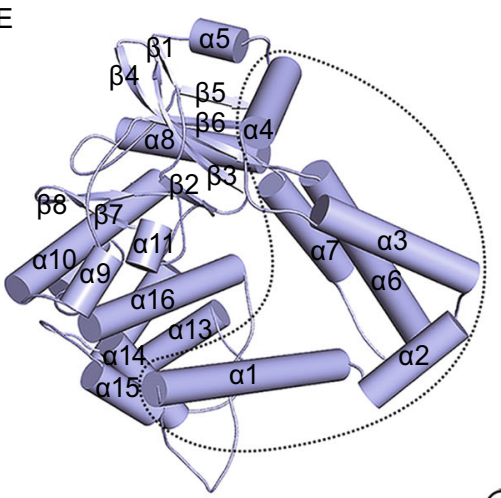

F
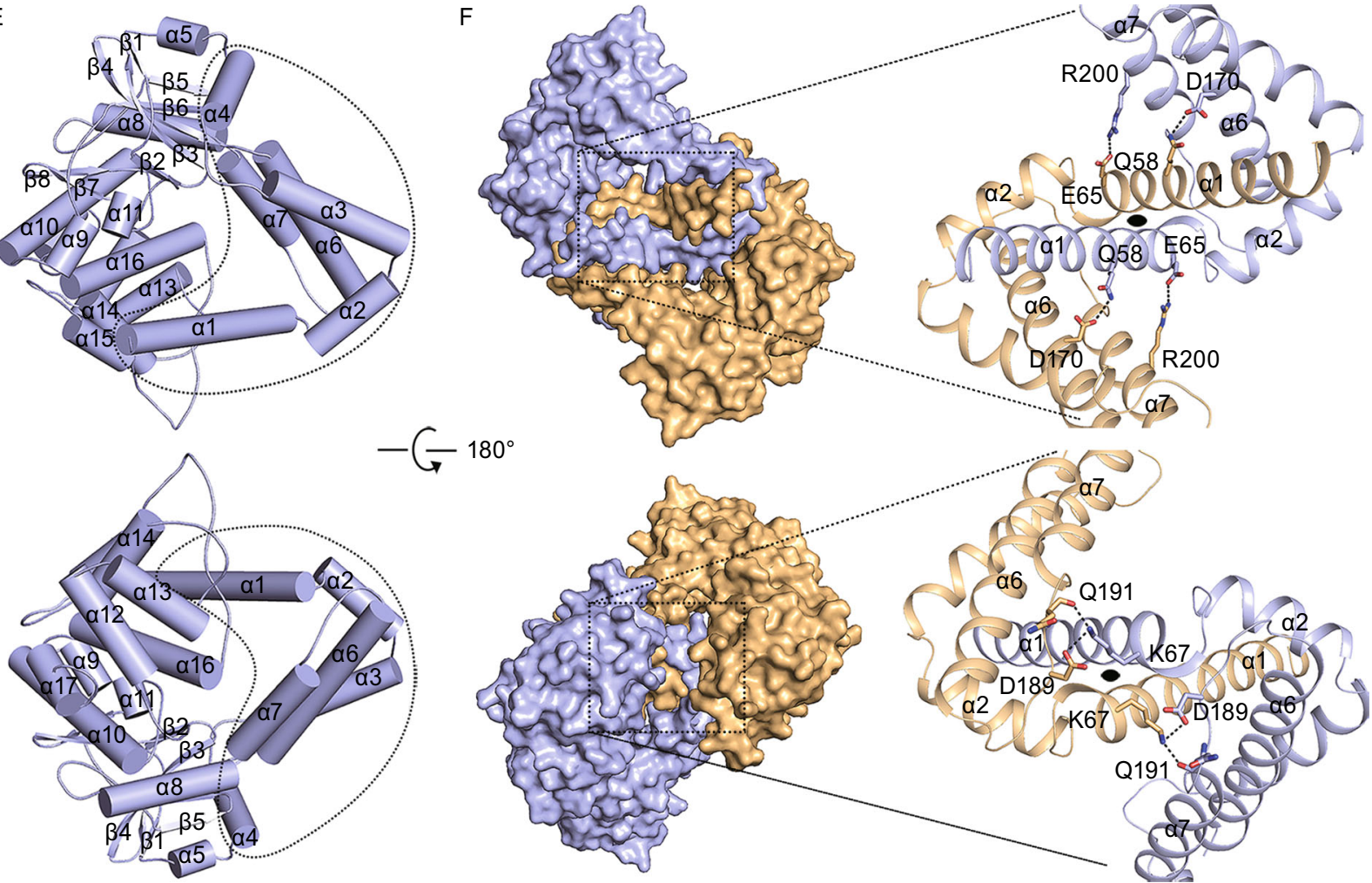

$180^{\circ}$

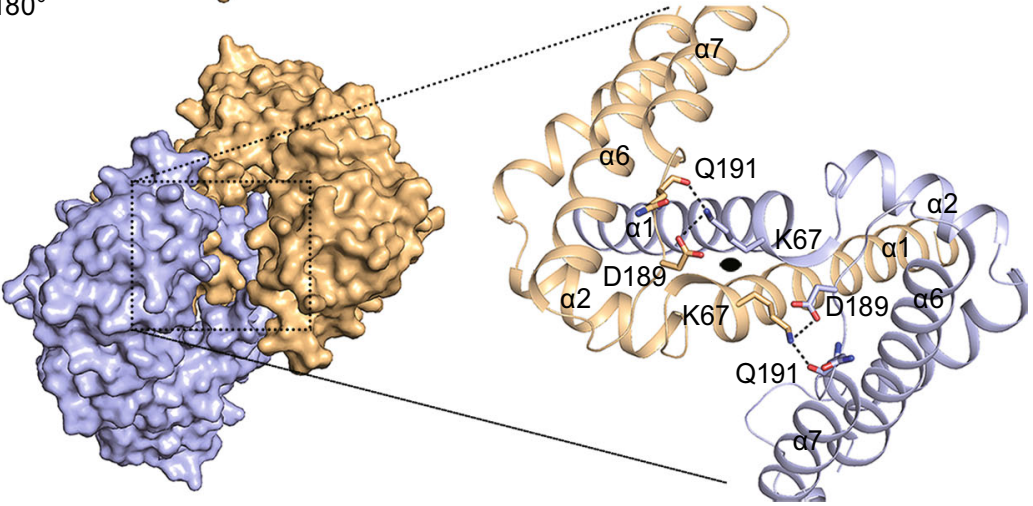

Figure 1. The functional and structural studies of Rv3197 (MABP-1). MABP-1 confers inducible resistance to erythromycin in mycobacteria (A-D). (A) mRNA levels of $r 3197$ in BCG determined by qRT-PCR after treatment with 6 mg/L erythromycin. (B) The growth of wild type BCG, $\Delta r$ 3197-BCG and pMV361-rv3197/ $r$ rv3197-BCG in 7H9 medium in the presence of $2 \mathrm{mg} / \mathrm{L}$ erythromycin. (C) Growth curves of pMV261-mc $\mathrm{m}^{2} 155$ and pMV261-rv3197- $\mathrm{mc}^{2} 155$ in $7 \mathrm{H} 9$ medium in the presence of $3 \mathrm{mg} / \mathrm{L}$ erythromycin. (D) Growth curves of pMV261-BCG and pMV261-rv3197-BCG in 7H9 medium in the presence of $4 \mathrm{mg} / \mathrm{L}$ erythromycin. All results are shown as the mean \pm standard deviation of three individual experiments. ${ }^{\star} P<0.05,{ }^{* \star} P<0.01,{ }^{* \star \star} P<0.001$. The crystal structure of MABP-1 (E-F). (E) The domain arrangement of a MABP-1 monomer. The accessory domain is encircled by the dashed outline. (F) Left, surface representation of the MABP-1 dimer. Right, the dimer subunit interface. Each subunit is coloured differently. The $\alpha 1$ and $\alpha 2$ helices from each subunit insert into each other's accessory domain. The black oval represents a two-fold axis perpendicular to the given view. Hydrogen bonds are shown as thick dashed lines.

gene (pMV261-rv3197-mc ${ }^{2} 155$ ) has a growth advantage over M. smegmatis that has an empty pMV261 vector (pMV261-mc ${ }^{2} 155$ ) (Fig. 1C). Likewise, the M. bovis BCG
pMV261-rv3197 construct also showed a growth advantage over wild type in the presence of $4 \mathrm{mg} / \mathrm{L}$ of erythromycin (Fig. 1D). In contrast, no growth difference was detected 
between pMV261-rv3197-mc ${ }^{2} 155$ and pMV261-mc ${ }^{2} 155$ and between pMV261-rv3197-BCG and PMV261-BCG in the absence of erythromycin (Fig. S1B and S1C). These data demonstrate that Rv3197 is a factor responsible for erythromycin resistance in both $M$. bovis and $M$. smegmatis. Based on sequence similarity, this protein is also expected to perform a similar role in other Mycobacteria (e.g., Mycobacterium tuberculosis, Mycobacterium kansasii, and Mycobacterium avium). In consideration of its antibiotic resistance phenotype, we henceforth refer to this protein as macrolide antibiotic binding protein-1 (MABP-1), signifying it as the first characterization of a macrolide antibiotic binding protein in Mycobacterium.

The crystal structure of apo-MABP-1 was determined at $2.2 \AA$ by single wavelength anomalous dispersion (SAD) (Table S2). MABP-1 is a homodimer with each subunit containing a bilobal kinase domain (Fig. S2) and an accessory domain (Figs. 1E and S2). According to a DALI search the kinase domain strongly resembles that of an ancient UbIB protein kinase (ADCK3) (Table S3). The accessory domain consists of six $\alpha$-helices: $\alpha 1-\alpha 4$ helices (residues 1120) and $\alpha 6-\alpha 7$ helices (residues 161-206). These regions are insertions that are not found in the canonical kinase structures. Crucially, the $\alpha 1$ and $\alpha 2$ helices in each subunit insert into the accessory domain of its adjoining subunit, resulting in an $\mathrm{N}$-terminal domain-swapped dimer (Fig. 1F). The accessible surface area that is buried upon dimerization is extensive, at $2021 \AA^{2}$ per subunit.

Structural analysis confirmed that MABP-1 has an ATP binding loop (P-loop) in its kinase domain suggesting ATP as a substrate. Indeed, a spectrophotometric assay shows that it can hydrolyse ATP $\left(k_{\mathrm{cat}}=0.0137 \mathrm{~s}^{-1}, K_{\mathrm{m}}=0.285 \mathrm{mmol} / \mathrm{L}\right)$ (Fig. S3A), values that are comparable to that for other ABC transporters (Lin et al., 2015). Moreover, our data showed that the presence of erythromycin does not affect the ATPase activity of MABP-1 (Fig. S3B). To gain further insights into how MABP-1 recognizes ATP, the crystal structures of MABP-1 in complex with AMPPNP and with ADP, were determined. Since AMPPNP and ADP bind to MABP-1 in a similar fashion, only the binding mode of AMPPNP (Figs. 2A and S4) is discussed below. In this complex (Figs. 2B and S5A), AMPPNP is stabilized by extensive hydrogen bonds and hydrophobic interactions. The amino acids like S139, K157, E204, E210, Q242, E243, W244, I245 and D304 play important roles in ATP binding (Supplementary Materials). The ATPase activity of these mutants is investigated. Compared with the wild-type enzyme, the S139A and E210A mutants lost $75 \%$ and $82 \%$ activity, respectively (Fig. 2C), emphasizing their importance in nucleotide binding. The E204A and Q242A mutants retained $85 \%$ and $83 \%$ activity, respectively and the W244A mutant retained full activity compared to the wild-type (Table S4), suggesting lesser roles for these residues. Importantly, the S139A, K157A, E204A, E210A, Q242A and W244A mutants all have a significant decreased erythromycin resistant phenotype in M. smegmatis (Fig. 2D), suggesting that antibiotic resistance also depends on the ATPase activity of MABP-1.

To understand how MABP-1 confers erythromycin resistance, the crystal structure of the complex was determined. The electron density for erythromycin was unequivocal revealing it is located in a shallow pocket formed by both subunits (Fig. 2E) where it is stabilized by extensive hydrogen bonds and hydrophobic interactions (Figs. 2E and S5B). Amongst these interactions are (i) a hydrogen bond between the carbonyl oxygen attached to $\mathrm{C} 9$ of the macrocyclic lactone ring (Fig. S6A) and the side-chain of K72 from the accessory domain of the opposing subunit, (ii) a hydrogen bond between the hydroxyl attached to $\mathrm{C} 12$ and the sidechain of D286, (iii) a hydrogen bond between the hydroxyl attached to $C 6$ and the side chain of E196, (iv) a hydrogen bond between the carbonyl oxygen attached to $\mathrm{C} 1$ and the side chain of R410 and (v) hydrophobic interactions involving A309, 1407 and M203 and the alkyl groups of the erythromycin (Fig. S5B). Two residues that contribute a significant portion of the surface of the erythromycin binding are E196 and R200, which are linked together by a salt bridge (Fig. 2E). As expected, the E65A, K72A, E196A, M203G, D286A, R410A mutants all significantly reduced the ability of MABP-1 to confer erythromycin resistance compared with wild-type $M$. smegmatis overexpressing MABP-1, emphasizing their critical roles in binding and in erythromycin resistance. In contrast, the R200A and I407G mutants produced only a slight reduction in the erythromycin resistance (Fig. 2F) while deletion of the entire $\mathrm{N}$-terminal 102 residues (Rv3197 ${ }^{\mathrm{N} \triangle 102}$ ) completely abolished drug resistance in M. smegmatis (Fig. 2F).

To investigate macrolide specificity of MABP-1, clarithromycin (14-membered ring) and azithromycin (15-membered ring) were also assessed for their ability to inhibit the growth of M. smegmatis (Figs. 2G and S7). The data showed that overexpression of MABP-1 results in greater resistance to both erythromycin and clarithromycin than azithromycin, suggesting that MABP-1 has a preference for 14-membered ring macrolides rather than those with 15 -membered rings (Supplementary Materials). The qRT-PCR data also shows that clarithromycin induces the upregulation of $r$ 3197 in BCG (Fig. 2H), again suggesting the 14-membered ring macrolides specificity of MABP-1.

In this study, we have identified a non-canonical $A B C$ protein encoded by the $r 3197$ gene in $M$. tuberculosis, as a macrolide antibiotic binding protein (MABP-1), which confers inducible resistance to the macrolide family of antibiotics. MABP-1 adopts an unusual three-dimensional fold with a kinase-like domain and a linked accessory domain. The accessory domain is indispensable for its function where it participates in dimerization of MABP-1 and in erythromycin binding. In addition, these data show that MABP-1 has a preference for 14-membered ring macrolides including erythromycin and clarithromycin rather than the 15-membered ring macrolides such as azithromycin. 

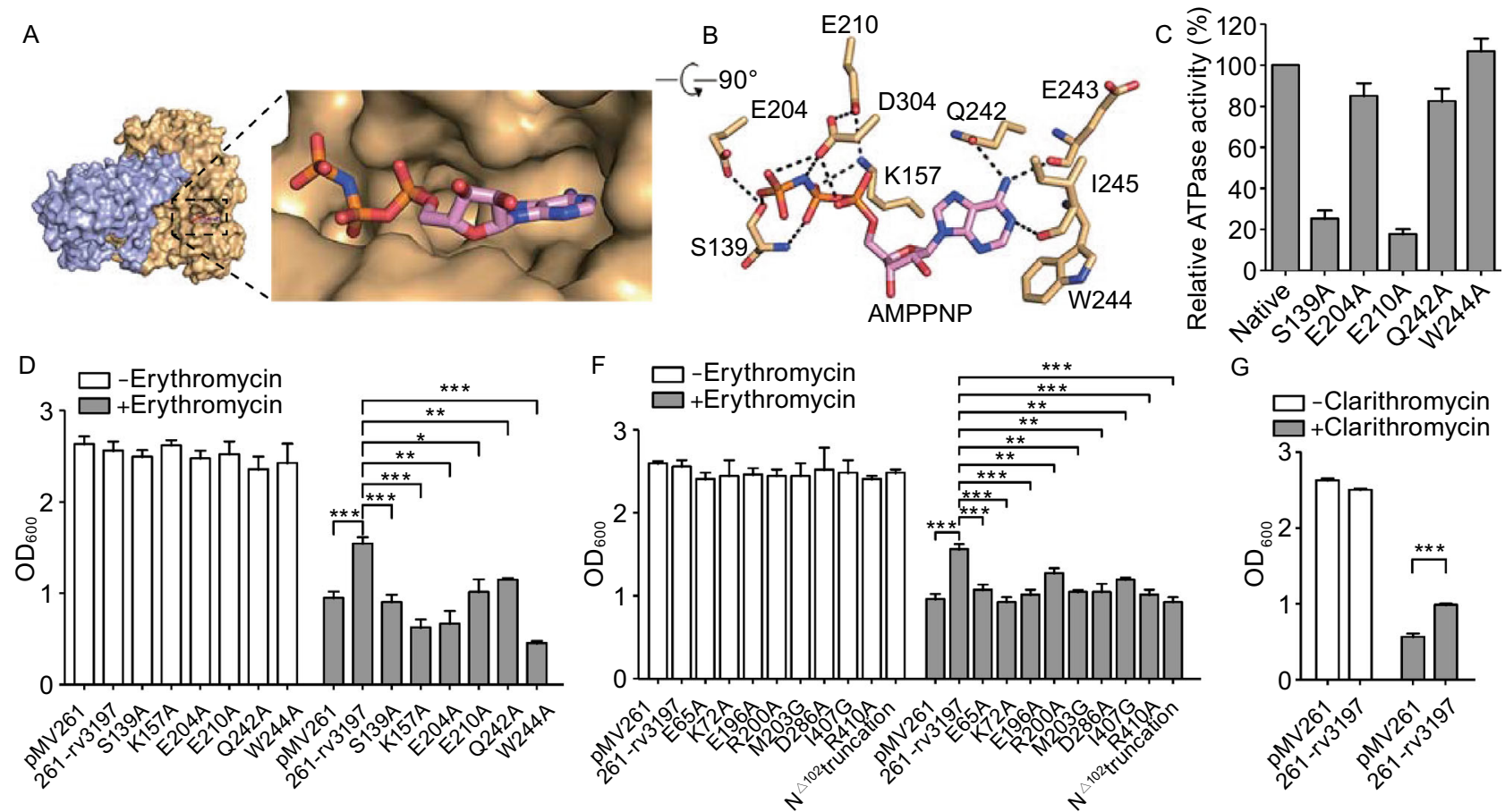

$\mathrm{G}$

$$
\square \text {-Clarithromycin }
$$

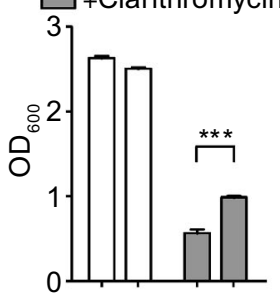

E
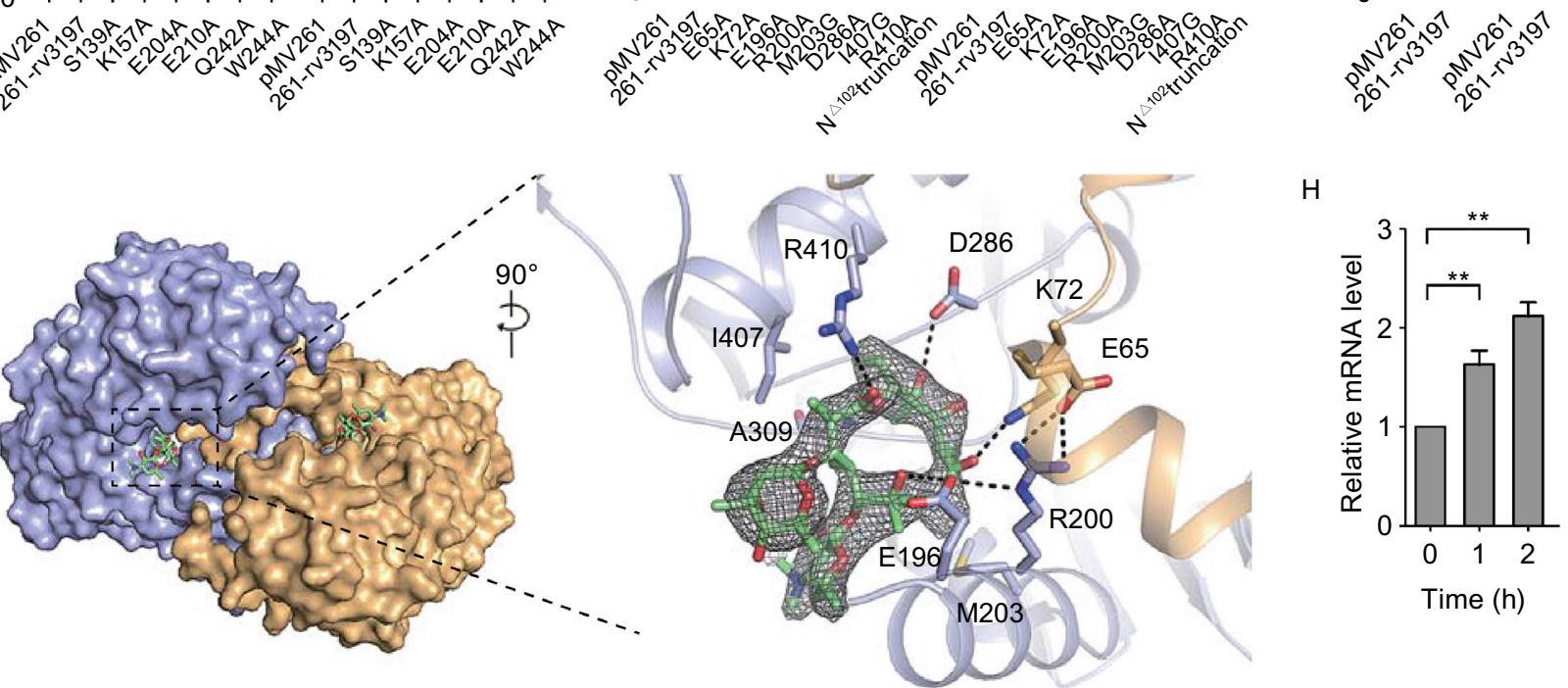

$\mathrm{H}$

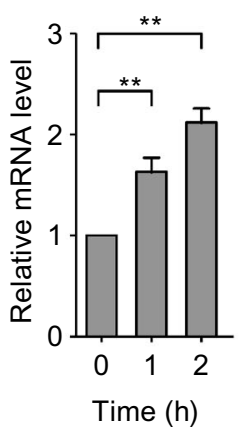

Figure 2. MABP-1 is an ATPase and the accessory domain of MABP-1 is vital for erythromycin binding and dimerization. (A) Structure of the MABP-1.AMPNP complex. MABP-1 is shown as a surface representation with AMPPNP as a stick model. Each subunit is coloured light orange and light blue. (B) The nucleotide-binding site in MABP-1. Hydrogen bonds are shown as dashed lines. (C) Relative ATPase activity of MABP-1 mutants in the nucleotide binding site (mean \pm SD of three individual experiments). (D) The growth of $\mathrm{mc}^{2} 155$ wild type and mutant strains in the presence (+) or absence (-) of $6.25 \mathrm{mg} / \mathrm{L}$ erythromycin (mean \pm SD of three individual experiments, ${ }^{*} P<0.05,{ }^{* *} P<0.01$, $\left.{ }^{* * *} P<0.001\right)$. (E) Left, surface representation of the erythromycin binding site. Erythromycin has green carbon atoms. Right, the $F_{o}-F_{c}$ omit map for erythromycin, contoured at $3.0 \sigma$. Residues that interact with erythromycin are shown as stick models. Hydrogen bonds are shown as dashed lines. (F) The growth of mc ${ }^{2} 155$ wild type and mutant strains in the presence (+) or absence (-) of $6.25 \mathrm{mg} / \mathrm{L}$ erythromycin. (G) The growth of pMV261-mc ${ }^{2} 155$ and pMV261-rv3197$\mathrm{mc}^{2} 155$ in $7 \mathrm{H} 9$ medium with (+) or without (-) $0.75 \mathrm{mg} / \mathrm{L}$ clarithromycin. (H) Quantification of mRNA levels of $r v 3197$ in BCG by qRT$\mathrm{PCR}$ at the indicated time points after treatment with $1.5 \mathrm{mg} / \mathrm{L}$ clarithromycin (mean $\pm \mathrm{SD}$ of three individual experiments, ${ }^{\star} P<0.05$, $\left.{ }^{\star *} P<0.01,{ }^{* * *} P<0.001\right)$.

Here we speculate that MABP-1 could dissociate erythromycin from the ribosome as has previously been suggested (Sharkey et al., 2016) in an ATP-dependent manner, reducing the concentration of erythromycin in the cytoplasm which is able to inhibit ribosomal activity. Another possibility is that MABP-1 is involved in modifying the structure of the antibiotic (Blair et al., 2015). The alternative hypothesis is that it cooperates with other efflux pumps to expel the drug 
(Nunez-Samudio and Chesneau, 2013). The structural comparison between MABP-1.AMPPNP and MABP-1.erythrmoycin may provide some clues to this hypothesis (Fig. S8 and Supplementary Materials). Clarification of the resistance mechanism of MABP-1 will require further investigation.

\section{FOOTNOTES}

We thank the staff at the Shanghai Synchrotron Radiation Facility (China) and Photon Factory (Japan) for their assistance in data collection. This work was supported by grants from the State Key Development Program for Basic Research of the Ministry of Science and Technology of China (973 Project Grant Nos. 2014CB542800), and the National Natural Science Foundation of China (Grant Nos. 81330036 and 81520108019 ). The atomic coordinates and structure factors have been deposited in the Protein Data Bank, www.pdb.org (PDB ID codes 5YJZ, 5YK0, 5YK1 and 5YK2).

Z.R. designed research. Q.Z., H.L., D.J., H.T. and B.Z. performed research. Q.Z., X.L., C.Y., H.Y., K.M. and Z.R. analyzed data. Q.Z., L.W.G., H.Y. and Z.R. wrote the paper. The authors declare no conflict of interest. This article does not contain any studies with human or animal subjects performed by the any of the authors.

Qingqing Zhang ${ }^{1,7}$, Huijuan Liu ${ }^{1}$, Xiang Liư ${ }^{2}$, Dunquan Jiang ${ }^{1}$, Bingjie Zhang ${ }^{3}$, Hongliang Tian ${ }^{4}$, Cheng Yang ${ }^{2}$, Luke W. Guddat ${ }^{5}$, Haitao Yang ${ }^{4}$, Kaixia $\mathrm{Mi}^{3}$, Zihe Rao ${ }^{1,2,6 凶}$

${ }^{1}$ College of Life Sciences, Nankai University, Tianjin 300071, China

2 College of Pharmacy, Nankai University, Tianjin 300071, China

${ }^{3}$ CAS Key Laboratory of Pathogenic Microbiology and Immunology, Institute of Microbiology, Chinese Academy of Sciences, Beijing 100101, China

${ }^{4}$ School of Life Sciences, Tianjin University, Tianjin 300072, China

${ }^{5}$ School of Chemistry and Molecular Biosciences, The University of Queensland, Brisbane, QLD 4072, Australia

${ }^{6}$ Laboratory of Structural Biology, School of Medicine, Tsinghua University, Beijing 100084, China

7 Tianjin International Joint Academy of Biotechnology \& Medicine, Tianjin 300457, China

$\bowtie$ Correspondence: raozh@nankai.edu.cn (Z. Rao)

\section{OPEN ACCESS}

This article is distributed under the terms of the Creative Commons Attribution 4.0 International License (http://creativecommons.org/ licenses/by/4.0/), which permits unrestricted use, distribution, and reproduction in any medium, provided you give appropriate credit to the original author(s) and the source, provide a link to the Creative Commons license, and indicate if changes were made.

Electronic supplementary material The online version of this article (https://doi.org/10.1007/s13238-017-0502-7) contains supplementary material, which is available to authorized users.

\section{REFERENCES}

Alekshun, M.N., and Levy, S.B. (2007). Molecular mechanisms of antibacterial multidrug resistance. Cell 128, 1037-1050.

Alimuddin Zumla, Andrew George, Virendra Sharma, Nick Herbert, and Masham, B. (2013). WHO's 2013 global report on tuberculosis: successes, threats, and opportunities. Lancet 382, 17651767.

Black, P.A., Warren, R.M., Louw, G.E., van Helden, P.D., Victor, T.C., and Kana, B.D. (2014). Energy metabolism and drug efflux in Mycobacterium tuberculosis. Antimicrob Agents Chemother 58, 2491-2503.

Blair, J.M., Webber, M.A., Baylay, A.J., Ogbolu, D.O., and Piddock, L.J. (2015). Molecular mechanisms of antibiotic resistance. Nat Rev Microbiol 13, 42-51.

Buriankova, K., Doucet-Populaire, F., Dorson, O., Gondran, A., Ghnassia, J.C., Weiser, J., and Pernodet, J.L. (2003). Molecular Basis of Intrinsic Macrolide Resistance in the Mycobacterium tuberculosis Complex. Antimicrob Agents Chemother 48, 143150.

Chen, S., Huai, P., Wang, X., Zhong, J., Wang, X., Wang, K., Wang, L., Jiang, S., Li, J., Peng, Y., et al. (2013). Risk factors for multidrug resistance among previously treated patients with tuberculosis in eastern China: a case-control study. Int J Infect Dis 17, e1116-e1120.

Dassa, E., and Bouige, P. (2001). The ABC of ABCs: a phylogenetic and functional classification of $A B C$ systems in living organisms. Res Microbiol 152, 211-229.

Dong, Y., Qiu, X., Shaw, N., Xu, Y., Sun, Y., Li, X., Li, J., and Rao, Z. (2015). Molecular basis for the inhibition of beta-hydroxyacylACP dehydratase HadAB complex from Mycobacterium tuberculosis by flavonoid inhibitors. Protein Cell 6, 504-517.

Günther, G. (2014). Multidrug-resistant and extensively drug-resistant tuberculosis: a review of current concepts and future challenges. Clin Med 14, 279-285.

Kerr, I. D., Reynolds, E.D., and Cove, J.H. (2005). ABC proteins and antibiotic drug resistance: is it all about transport? Biochem Soc 33, 1000-1002.

Lin, D.Y., Huang, S., and Chen, J. (2015). Crystal structures of a polypeptide processing and secretion transporter. Nature 523, 425-430.

Nunez-Samudio, V., and Chesneau, O. (2013). Functional interplay between the ATP binding cassette $\operatorname{Msr}(D)$ protein and the membrane facilitator superfamily $\operatorname{Mef}(\mathrm{E})$ transporter for macrolide resistance in Escherichia coli. Res Microbiol 164, 226-235.

Shim, T.S., and Jo, K.-W. (2013). Medical treatment of pulmonary multidrug-resistant tuberculosis. Infect Chemother 45, 367-374.

Sharkey, L.K., Edwards, T.A., and O'Neill, A.J. (2016). ABC-F proteins mediate antibiotic resistance through ribosomal protection. MBio 7, e01975.

WHO (2014). Global tuberculosis report 2014 (Geneva, World Health Organization). 